\title{
First-Principles Investigations to Evaluate the Spin-Polarized Metal-to-Insulator Transition of Halide Cuprite Perovskites for Smart Windows
}

\author{
June Ho Lee ${ }^{\dagger}$, Seong Hun Kim ${ }^{\dagger}$, Kyung-Yeon Doh ${ }^{\dagger}$, Eun Ho Kim ${ }^{\dagger}$, Donghwa Lee*,†,
}

\begin{abstract}
${ }^{\dagger}$ Department of Materials Science and Engineering (MSE), and Division of Advanced Materials Science (AMS), Pohang University of Science and Technology (POSTECH), Pohang 37673, Republic of Korea

¥Institute for Convergence Research and Education in Advanced Technology (I_CREATE), Yonsei University, Incheon 21983,

Republic of Korea
\end{abstract}




\section{Methodology}

First-principle density functional theory (DFT) calculations were performed using Vienna ab initio simulation package (VASP) ${ }^{1,2}$. To obtain ground state structure, the DFT calculations were conducted on the basis of the Perdew-Burke-Ernzerhof revised for solids of generalized gradient approximation (PBEsol-GGA) ${ }^{3}$ implemented with projector augmented-wave (PAW) pseudopotential ${ }^{4}$. To consider the effect of the van der Waals ( $\mathrm{vdW}$ ) interaction between A-site and X-site halogen, we adopted internal parameters of the Grimme's DFT-D3 vdW 5 as implemented in VASP code. A energy cutoff of $550 \mathrm{eV}$ with the Monkhorst-Pack k-point grid ${ }^{6}$ of $8 \times 8$ $\mathrm{x} 4$ at the $\Gamma$-point was used. All structure were fully relaxed until the Hellmann-Feynman forces on them were less than 0.005 $\mathrm{eV} \cdot A^{-1}$.

To correct the well-known bandgap underestimation problem of DFT, we used $\mathrm{G}_{0} \mathrm{~W}_{0}$ method based on the wavefunction from PBEsol (PBEsol $+\mathrm{G}_{0} \mathrm{~W}_{0}$ ) for the bandgap calculation. To identify convergence criteria of $\mathrm{G}_{0} \mathrm{~W}_{0}$, we carried out calculations for $\mathrm{Cs}_{2} \mathrm{CuCl}_{4}$ with 4 parameters (the number of frequency points (NOMEGA), energy cutoff (ENCUT), energy cutoff for response function (ENCUTGW) and the number of band (NBANDS)). Our study identified that NOMEGA $=128$, ENCUT $=550$, ENCUTGW $=300$ and NBANDS $=352$ are sufficient conditions for the $\mathrm{G}_{0} \mathrm{~W}_{0}$ bandgap convergence; the variation of $\mathrm{G}_{0} \mathrm{~W}_{0}$ bandgap is within $0.02 \mathrm{eV}$.

Table S1. Convergence test of $\mathrm{G}_{0} \mathrm{~W}_{0}$ bandgap of $\mathrm{Cs}_{2} \mathrm{CuCl}_{4}$.

\begin{tabular}{cccc|c|ccc}
\hline NOMEGA & 128 & 144 & 180 & ENCUT (eV) & 450 & 550 & 650 \\
Band gap (eV) & 1.607 & 1.582 & 1.600 & Band gap (eV) & 1.597 & 1.607 & 1.618 \\
\hline & & & & & & & \\
\hline
\end{tabular}

For the study, bandgaps of the $\mathrm{A}_{2} \mathrm{CuX}_{4}(\mathrm{~A}: \mathrm{K}, \mathrm{Rb}, \mathrm{Cs} ; \mathrm{X}: \mathrm{Cl}, \mathrm{Br}, \mathrm{I})$ systems are calculated by using $\mathrm{G}_{0} \mathrm{~W}_{0}$ method. Then, PBEsol $+\mathrm{U}^{7}$ calculations using on-site coulomb $\mathrm{U}$ and exchange $\mathrm{J}$ term are performed to obtain band structure, density of states and bandgap of hybrid $\left(\mathrm{MA}_{2} \mathrm{CuCl}_{4}, \mathrm{EA}_{2} \mathrm{CuCl}_{4}\right)$ and strained systems (compressive and tensile stained $\mathrm{Rb}_{2} \mathrm{CuBr}_{4}$ ) since the converged electronic structures from $\mathrm{G}_{0} \mathrm{~W}_{0}$ calculations are computationally too expensive. The parameters ( $\mathrm{U}$ and $\mathrm{J}$ ) are chosen based on $\mathrm{G}_{0} \mathrm{~W}_{0}$ bandgap. Table $\mathrm{S} 2$ show the comparison on the bandgap between $\mathrm{G}_{0} \mathrm{~W}_{0}$ and PBEsol+U methods.

Table S2. Comparison of bandgap using $\mathrm{G}_{0} \mathrm{~W}_{0}$ and PBEsol+U for the $\mathrm{A}_{2} \mathrm{CuX}_{4}(\mathrm{~A}: \mathrm{Cs}, \mathrm{Rb}, \mathrm{K} ; \mathrm{X}: \mathrm{Cl}, \mathrm{Br}, \mathrm{I})$.

\begin{tabular}{c|ccc|ccc|ccc}
\hline & \multicolumn{3}{|c}{$\mathrm{Cl}$} & \multicolumn{3}{c|}{$\mathrm{Br}$} & \multicolumn{3}{c}{$\mathrm{I}$} \\
& $\mathrm{Cs}$ & $\mathrm{Rb}$ & $\mathrm{K}$ & $\mathrm{Cs}$ & $\mathrm{Rb}$ & $\mathrm{K}$ & $\mathrm{Cs}$ & $\mathrm{Rb}$ & $\mathrm{K}$ \\
\hline $\mathrm{G}_{0} \mathrm{~W}_{0}$ & $\mathbf{1 . 6 1}$ & $\mathbf{1 . 2 9}$ & $\mathbf{1 . 0 2}$ & $\mathbf{1 . 1 1}$ & $\mathbf{0 . 8 1}$ & $\mathbf{0 . 6 1}$ & $\mathbf{0 . 5 2}$ & $\mathbf{0} .27$ & metal \\
PBEsol $+\mathrm{U}$ & 1.61 & 1.38 & 1.18 & 1.03 & 0.82 & 0.63 & 0.49 & 0.29 & metal \\
\hline Fitting parameter & \multicolumn{6}{c|}{$\mathrm{U}: 10.1, \mathrm{~J}: 5$} & & & \multicolumn{3}{c}{$\mathrm{U}: 11.5, \mathrm{~J}: 5$} \\
\hline
\end{tabular}

Our calculation results predict that the Hubbard correction term $(\mathrm{U}: 10.1 \mathrm{eV}, \mathrm{J}: 5 \mathrm{eV}$ for chloride and bromide system, $\mathrm{U}: 11.5 \mathrm{eV}$, $\mathrm{J}: 5 \mathrm{eV}$ for iodide system) can successfully reproduce the bandgap of $\mathrm{G}_{0} \mathrm{~W}_{0}$ method. Thus, we adapt PBEsol+U calculations to obtain the bandgaps of various $\mathrm{A}_{2} \mathrm{CuX}_{4}$ systems.

The single shot quasi-particle energies calculation $\left(\mathrm{G}_{0} \mathrm{~W}_{0}\right)$ were performed to demonstrate the bandgap reduction, since it is one of the known methods to predict the transition between insulating phase and metallic phase by using the first-principles calculations. Wegkamp D. et al., demonstrate that the bandgap of monoclinic $\mathrm{VO}_{2}$ can collapse under doping concentration of 0.075 holes per one $\mathrm{V}$ atom by using the first-principles calculations ${ }^{8}$. 


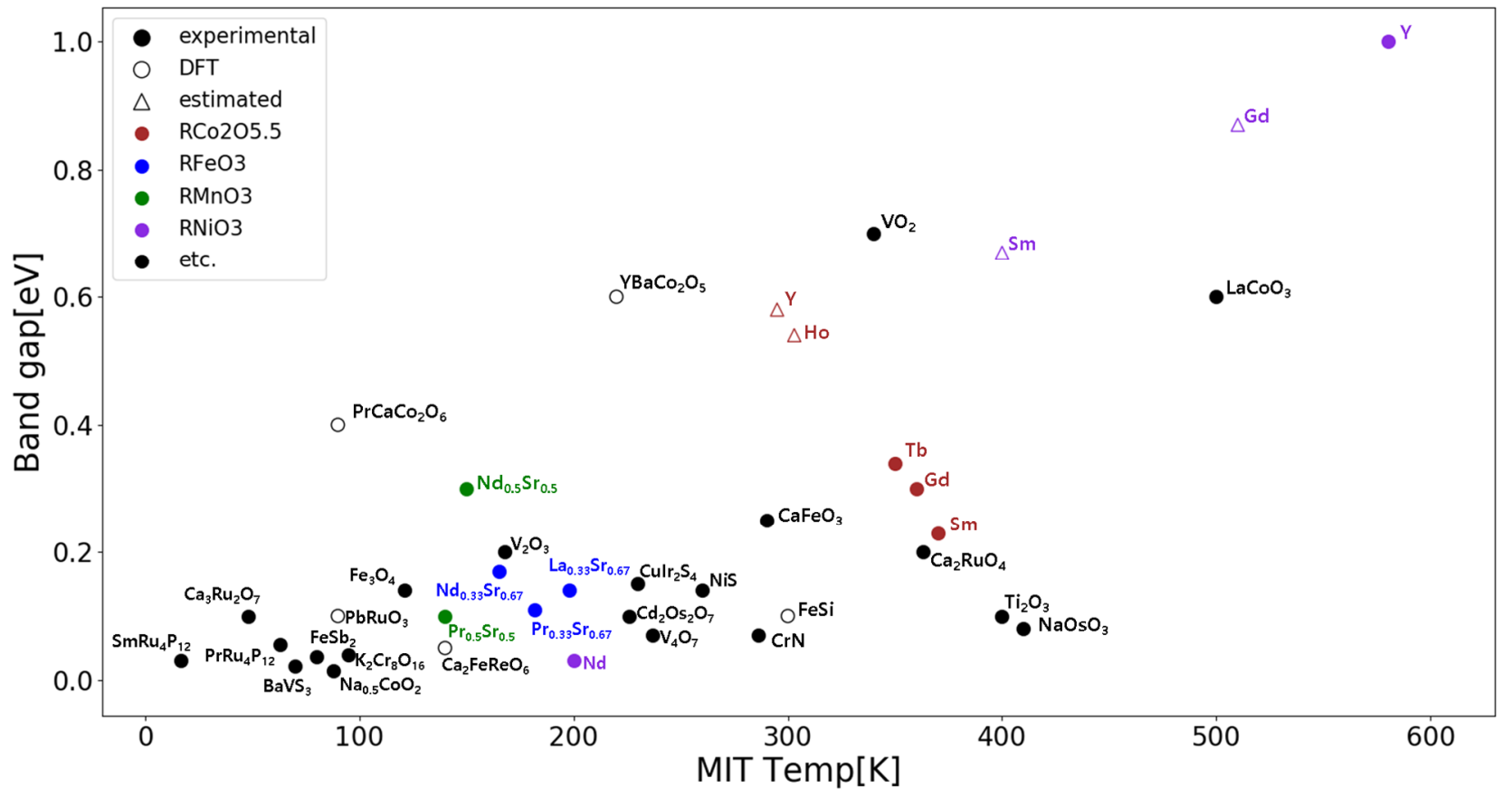

Supplementary Figure 1. Bandgap vs transition temperature of various metal insulator transition materials. Filled circle: experimental results; open circle: DFT calculation results; open triangle: extrapolating results from same metal-oxide system. Additionally, we categorized each data into Cobaltite (Brown circle), Ferrite (Blue circle), Manganite (green circle) and Nickelate (Magenta circle) system. 


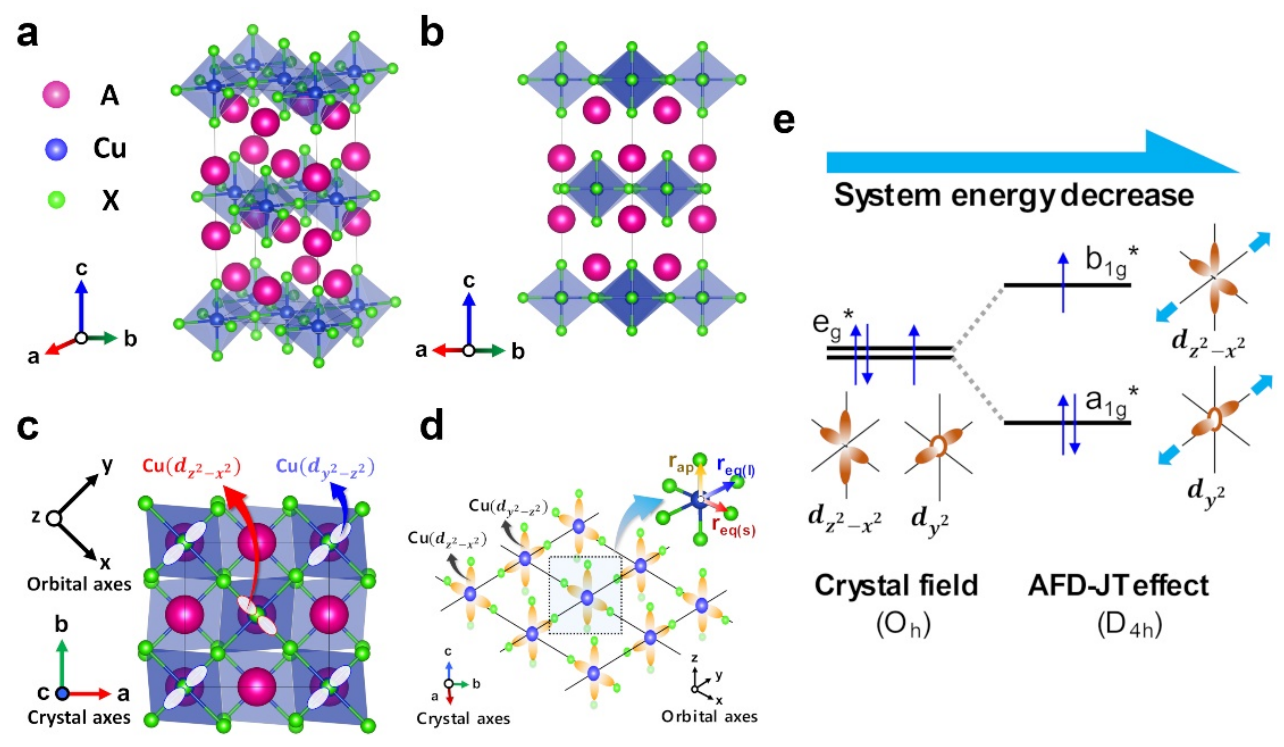

Supplementary Figure 2. Crystal structure of halide cuprite layered perovskites $\left(\mathrm{A}_{2} \mathrm{CuX}\right.$ ) views (a) from side, (b) along 110 direction and (c) along 001 direction with singly occupied electron on each $\mathrm{Cu}$ site. (d) Schematic figure for the Anti-ferrodistortive Jahn-Teller (AFD-JT) distortion originated from orbital ordering (between $\mathrm{Cu}-d_{z^{2}-x^{2}}$ and $\mathrm{Cu}-d_{y^{2}-z^{2}}$ ). a, b and c represent the crystallographic axes; $x, y$ and $z$ represent the axes of the orbitals. We represent three bond lengths in the $\mathrm{CuX}_{6}$ octahedron. (inset of figure S2d) Apical bond length $r_{a p}$ is the bond along the out-of-plane direction [001], equatorial short bond length $r_{\text {eq(s) }}$ and equatorial long length $\mathrm{r}_{\mathrm{eq}(\mathrm{l})}$ are short and long bond lengths along the in-plane direction, respectively. The color sphere depict the Asite atom (magenta), $\mathrm{Cu}$ (blue) and X-site halogen (green). (e) Schematic figure about origin of AFD-JT distortion. We only represent $\mathrm{e}_{\mathrm{g}} *$ orbitals $\left(\mathrm{b}_{1 \mathrm{~g}} *\right.$ and $\left.\mathrm{a}_{1 \mathrm{~g}}{ }^{*}\right)$ formed by crystal field splitting (AFD-JT distortion).

\section{Anti-ferrodistortive Jahn-Teller (AFD-JT) distortion}

In the layered halide cuprite $\left(\mathrm{A}_{2} \mathrm{CuX} \mathrm{X}_{4}\right)$, Jahn-teller active $\mathrm{Cu}$ atom have odd number $\left(\mathrm{d}^{9}\right)$ of electron. Therefore, three electrons occupy $\mathrm{e}_{\mathrm{g}}{ }^{*}$ (antibonding $\mathrm{e}_{\mathrm{g}}$ states) state. In this electron configuration, total energy of system can be reduced by AFD-JT distortion breaking the degeneracy of $\mathrm{e}_{\mathrm{g}}{ }^{*}$ states to $\mathrm{b}_{1 \mathrm{~g}} *$ and $\mathrm{a}_{1 \mathrm{~g}} *$. In the aspect of bonding length, doubly occupied antibonding $\mathrm{a}_{1 \mathrm{~g}} *$ state reduce bonding character, thus has long bonding length. On the other hand, singly occupied $\mathrm{b}_{1 \mathrm{~g}} *$ cannot sufficiently reduce bonding character, so shows short bonding length. As a result, identical equatorial bonding length $\left(\mathrm{r}_{\mathrm{eq}}\right)$ in Non-JT system is divided into longer $\left(\mathrm{r}_{\mathrm{eq}(\mathrm{l})}\right)\left(\right.$ from $\left.\mathrm{a}_{1 \mathrm{~g}}{ }^{*}\right)$ and shorter $\left(\mathrm{r}_{\mathrm{eq}(\mathrm{s})}\right)$ (from $\left.\mathrm{b}_{1 \mathrm{~g}}{ }^{*}\right)$ ones. 
a

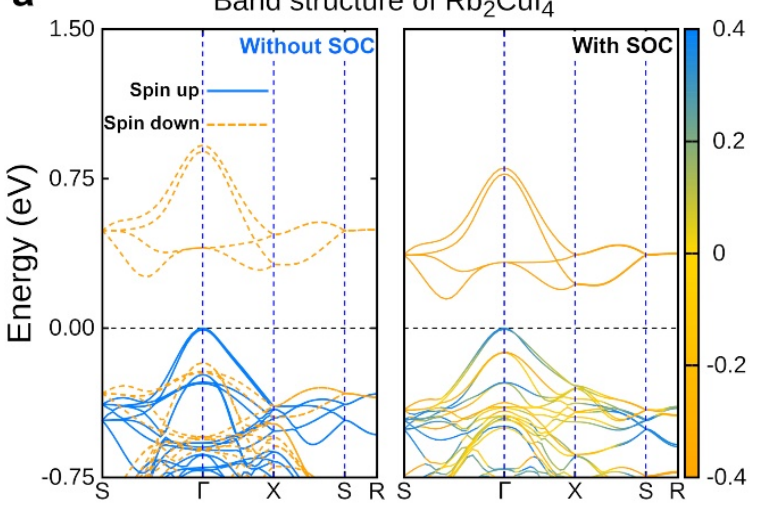

b

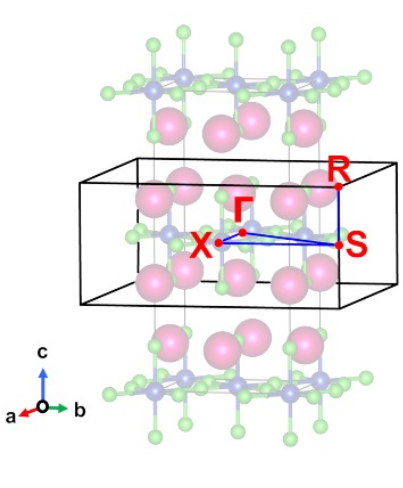

C

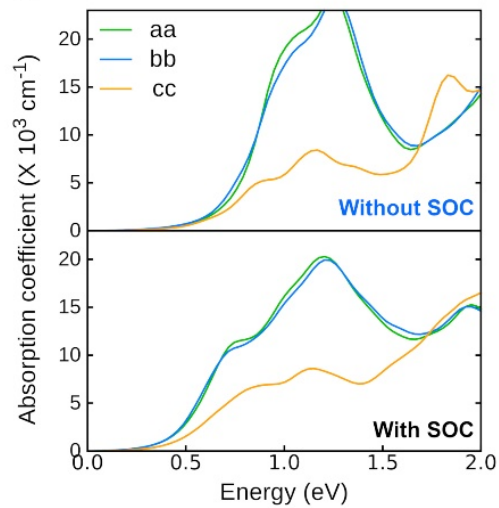

Supplementary Figure 3. (a) Calculated band structure of $\mathrm{Rb}_{2} \mathrm{CuI}_{4}$ without and with spin-orbit coupling (SOC) effect and (b) schematic view of high symmetric k-points. (c) Calculated absorption spectra using PBEsol $+\mathrm{U}$ for $\mathrm{Rb}_{2} \mathrm{CuI}_{4} \mathrm{Without}$ and with SOC effect. 

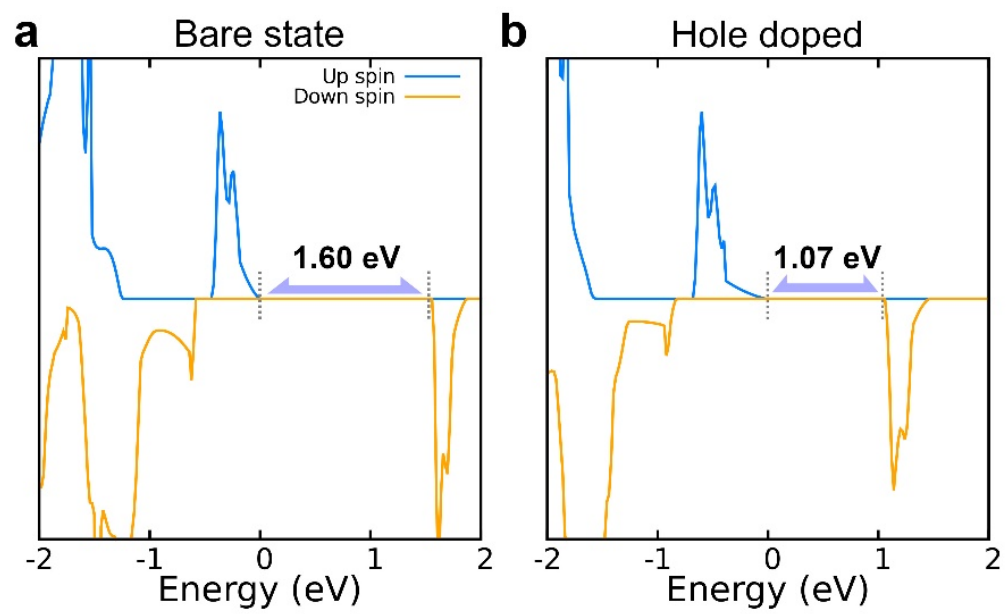

Supplementary Figure 4. Total density of states for the (a) bare and (b) hole doped $\mathrm{Cs}_{2} \mathrm{CuCl}_{4}$ by using single shot quasi-particle $\mathrm{GW}\left(\mathrm{G}_{0} \mathrm{~W}_{0}\right)$ calculation which can reproduce hole-driven bandgap reduction. Doping level is 0.075 per $\mathrm{Cu}$ atom which is corresponding to the hole density for the hole-driven metallic transition in $\mathrm{VO}_{2}$ structure. We can see the hole induced bandgap reduction from bare $(1.60 \mathrm{eV})$ to hole-doped $(1.07 \mathrm{eV})$ system. 
a

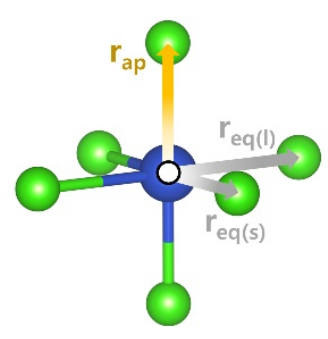

b

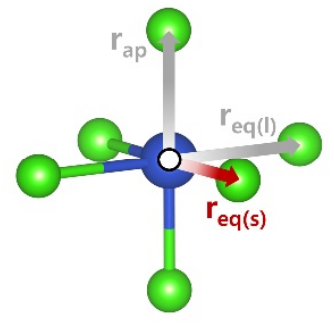

C

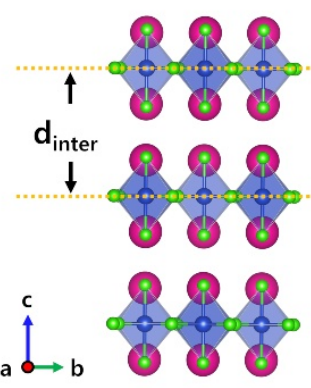

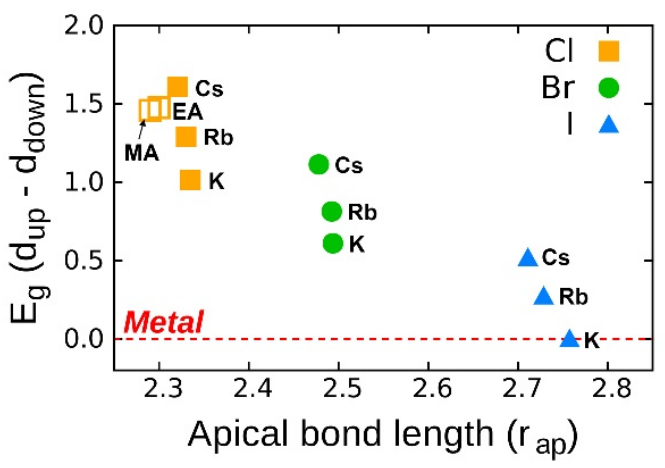
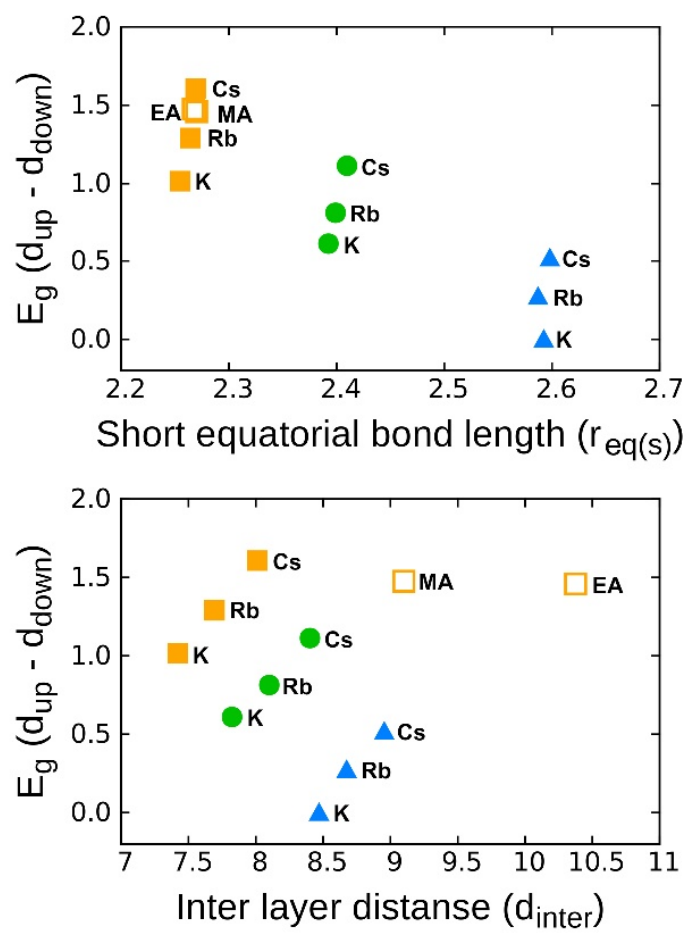

Supplementary Figure 5. Spin-polarized bandgap as a function of (a) Apical banding length ( $\mathrm{r}_{\mathrm{ap}}$ ), (b) equatorial short bonding length $\left(\mathrm{r}_{\mathrm{eq}}(\mathrm{s})\right.$ ) and (c) inter-layer distance $\left(\mathrm{d}_{\text {inter }}\right)$ for the $\mathrm{A}_{2} \mathrm{CuX}_{4}$ systems. Figures on the left show graphical representations of each structural parameter. $\mathrm{G}_{0} \mathrm{~W}_{0}$ method were performed to get bandgap. 
a

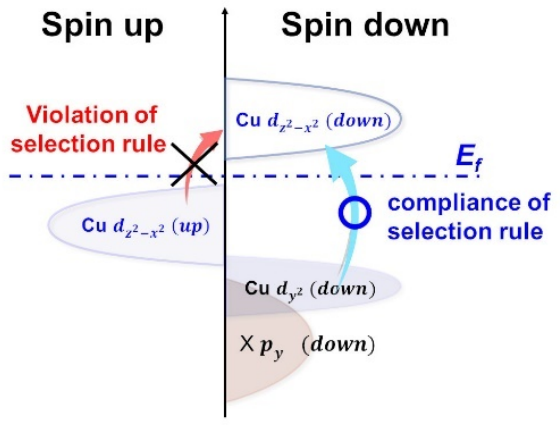

Spin polarized insulator b

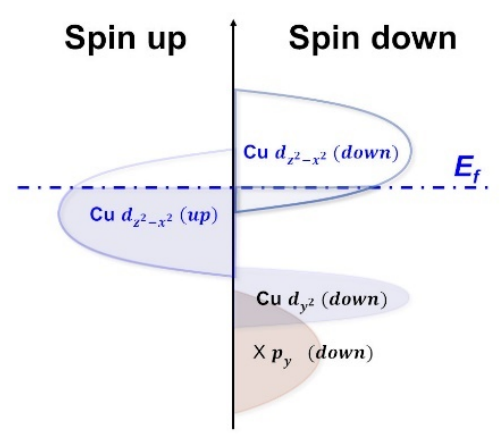

Spin polarized metal

Supplementary Figure 6. Schematic PDOS of (a) spin polarized insulator and (b) spin polarized metal which can show main idea for the smart window in this study. In the case of low temperature insulating phase, optical transition from spin-polarized bandgap is suppressed due to the violation of selection rule. As a result, optical transition occurs from relatively larger bandgap which can obey selection rule. In the higher temperature metallic case, spin polarized bandgap can be easily closed by hole-driven bandgap reduction because of their smaller size of bandgap. 


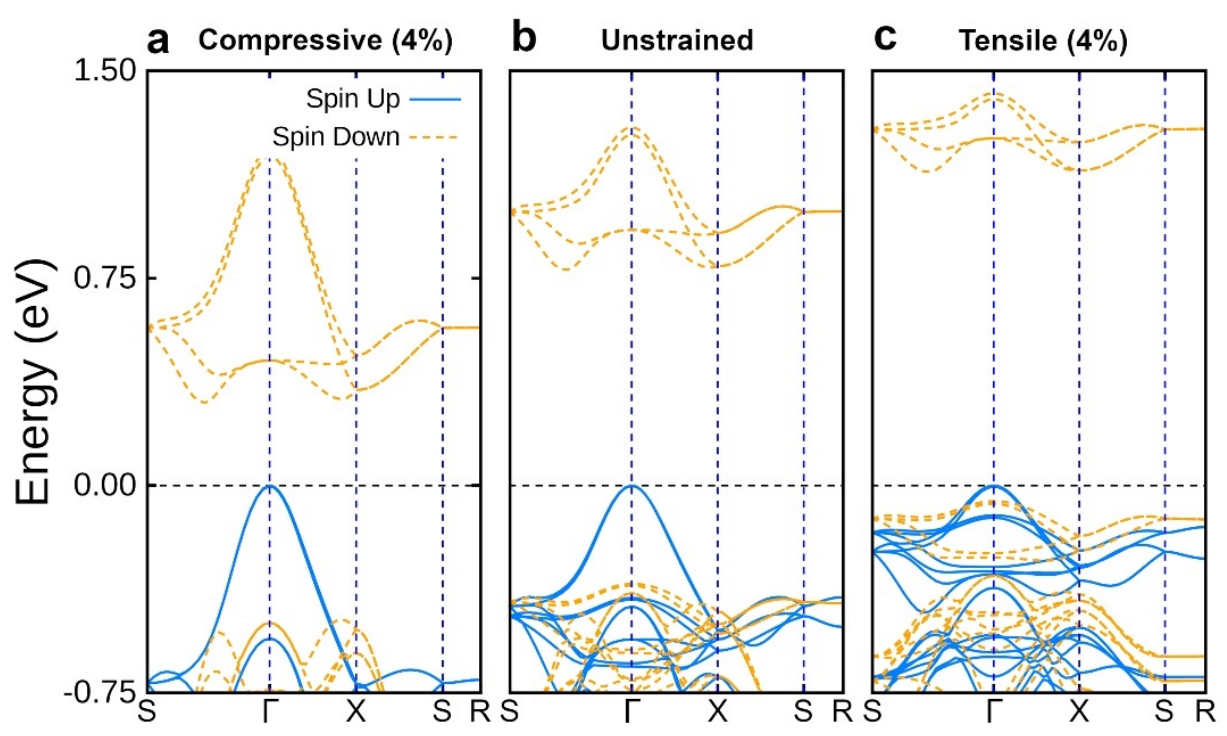

Supplementary Figure 7. Electronic band structure of $\mathrm{Rb}_{2} \mathrm{CuBr}_{4}$ under external bi-axial (a) $4 \%$ compressive strain, (b) unstrained and (c) 4\% tensile strain using PBEsol + U. Blue solid (orange dash) line indicates the up (down) spin.

In other to verify the spin-polarized bandgap changes according to antiferrodistortive Jahn-Teller (AFD-JT) distortion, we calculated the band structure under external bi-axial strains on $\mathrm{Rb}_{2} \mathrm{CuBr}_{4}$. For the compressive strained case, we can clearly see that the increase in AFD-JT distortion improves the dispersion of valence and conduction band and so reduces the gap between spin-up and spin-down band (Fig. S7a). For the tensile strained case, the reduced AFD-JT distortion decreases the dispersion of valence and conduction band and so increases the bandgap (Fig. S7c). These results are exactly coincident with A-site exchange effect described in main manuscript. 


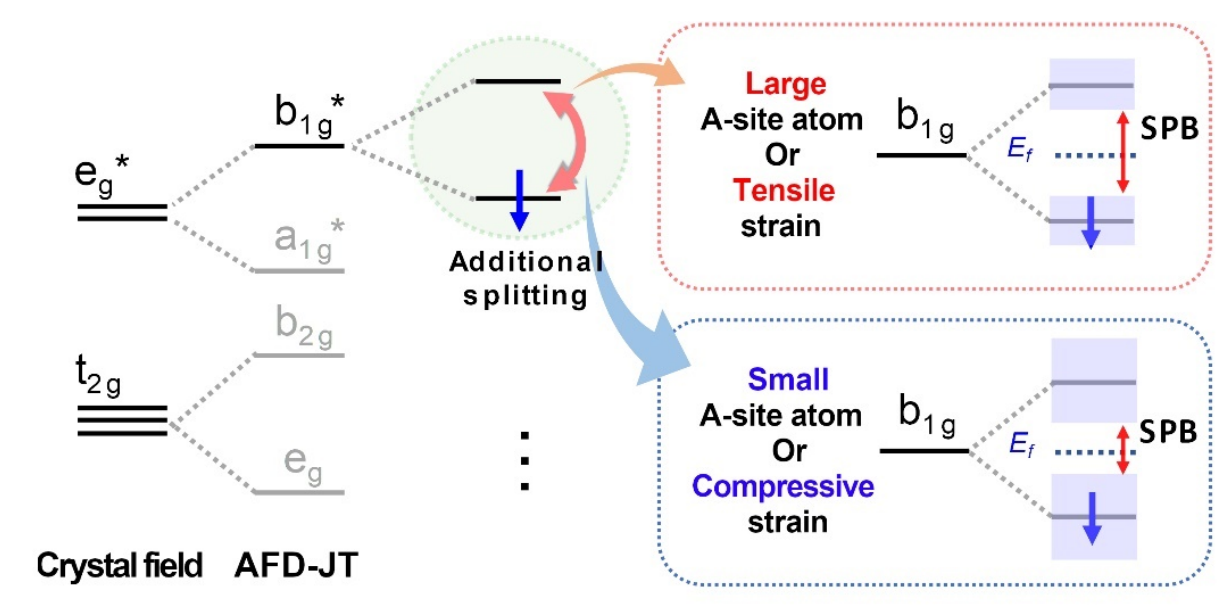

Supplementary Figure 8. Schematic view of molecular orbital diagram for each splitting mechanism. From the Crystal field, degenerated d orbital of $\mathrm{Cu}$ split in to the $\mathrm{e}_{\mathrm{g}}$ and $\mathrm{t}_{2 \mathrm{~g}}$ states. Additionally, due to the antiferrodistortive Jahn-teller effect (AFD-JT), $\mathrm{e}_{\mathrm{g}}$ states is splitting into $b_{1 g}$ and $a_{1 g}$ states, while $t_{2 g}$ state is splitting into $b_{2 g}$ and $e_{g}$ states, respectively. Finally, orbital ordering cause additional spin splitting of $\mathrm{Cu}^{2+}$ ion. Single electron occupies splitted low spin level. As a result, the energy difference between singly occupied spin state and unoccupied spin state becomes a spin polarized bandgap in the $\mathrm{A}_{2} \mathrm{CuX}$ layered halide perovskite. Larger A-site atom or tensile strain that can improve AFD can increase the bandgap due to the decrease of band dispersion. Smaller A-site cation or compressive strain tend to be opposite.

Increase in the size of A-site strengthens the structural antiferrodistortive Jahn-teller (AFD-JT) distortion by pushing away the $\mathrm{CuX}_{6}$ octahedrons. The strengthened AFD-JT distortion increase the bandgap by decreasing the band dispersion. This is equivalent to the change in bandgap under tensile strain. On the contrary, decrease in the size of A-site weakens the structural antiferrodistortive Jahn-teller (AFD-JT) distortion by pulling the $\mathrm{CuX}_{6}$ octahedrons together. As a result, the weakened AFD-JT distortion decrease the bandgap by increasing the band dispersion. This is exactly the same to the change in bandgap under compressive strain. 

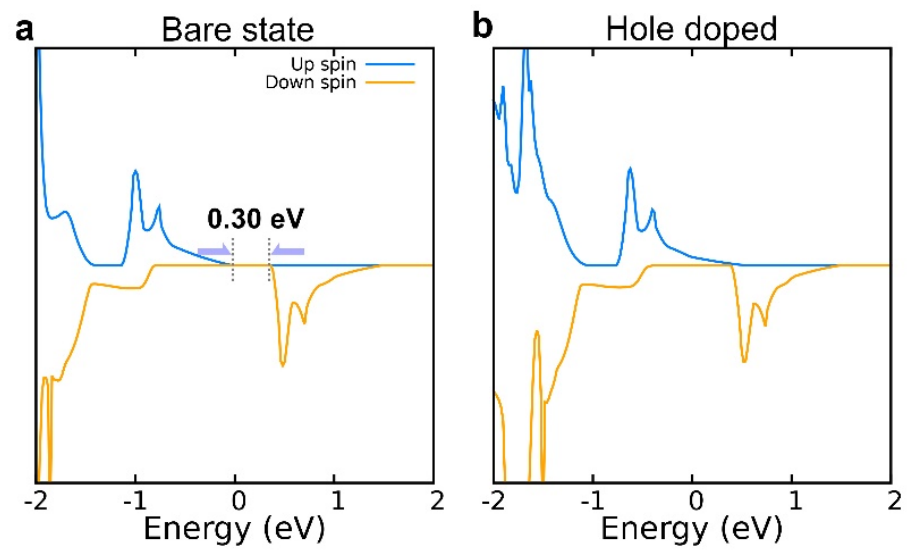

Supplementary Figure 9. Total density of states calculation for the (a) bare and (b) hole doped $\mathrm{Rb}_{2} \mathrm{CuBr}_{4}(4 \%$ compressive strained) by using single shot quasi-particle $\mathrm{GW}\left(\mathrm{G}_{0} \mathrm{~W}_{0}\right)$. Doping level is 0.075 per $\mathrm{Cu}$ atom which is corresponding to the hole density for the hole-driven metallic transition in $\mathrm{VO}_{2}$ structure.

Since bulk modules of $\mathrm{Rb}_{2} \mathrm{CuCl}_{4}(17.1 \mathrm{GPa})^{9}$ is lower than that of other oxide systems $\left(\mathrm{VO}_{2}: 140 \mathrm{GPa}, \mathrm{ZnO}: 183 \mathrm{GPa}, \mathrm{SnO} 2: 205\right.$ $\mathrm{GPa}$,), the control of the spin-polarized bandgap of $\mathrm{A}_{2} \mathrm{CuX}_{4}$ is expected to be relatively easier. 


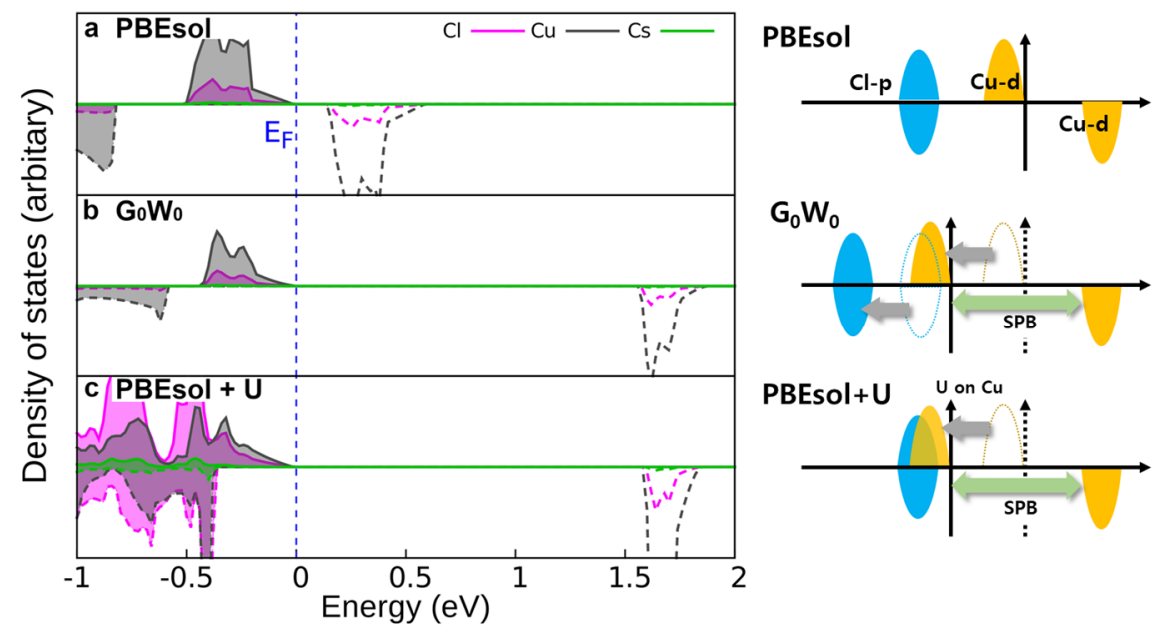

Supplementary Figure 10. Partial density of states of the $\mathrm{Cs}_{2} \mathrm{CuCl}_{4}$ by using (a) PBEsol, (b) $\mathrm{G}_{0} \mathrm{~W}_{0}$ and (c) PBEsol+U. In the PBEsol method, bandgap was calculated too small $(0.15 \mathrm{eV})$ because of the well-known bandgap underestimation problem of density functional theory calculations. To correct this issue, we also calculate the bandgap with $\mathrm{G}_{0} \mathrm{~W}_{0}$ and $\mathrm{PBEsol}+\mathrm{U}$ ( $\mathrm{U}: 10.1$ $\mathrm{eV}, \mathrm{J}=5 \mathrm{eV}$ ) methods.

Partial density of states (PDOS) obtained using PBEsol and $\mathrm{G}_{0} \mathrm{~W}_{0}$ show a similar shape except spin polarized bandgap of PBEsol is much smaller than that of $\mathrm{G}_{0} \mathrm{~W}_{0}$. On the other hand, PBEsol+ $\mathrm{U}$ calculation yields a bandgap equal to $\mathrm{G}_{0} \mathrm{~W}_{0}$ by shifting the valence band made by $\mathrm{Cu}-\mathrm{d}_{\text {up }}$ orbitals to lower energy state due to the $\mathrm{U}$ term applied to $\mathrm{Cu}$. However, PBEsol+U do not shift Cl-p orbitals, which is supposed to be shifted to a lower energy state, as observed in $\mathrm{G}_{0} \mathrm{~W}_{0}$ result. As a result, the lower shifted Cu- $\mathrm{d}_{\text {up }}$ orbitals partially overlap with Cl-p band and so the lower part of the valence band of PBEsol+U shows the density of misplaced Cl-p orbitals. This pattern was also observed for $\mathrm{Br}$ and I cases. However, frontier orbitals ( $\mathrm{CBM}: \mathrm{Cu}-\mathrm{d}_{\mathrm{down}}, \mathrm{VBM}$ : hybridization of Cu- $\mathrm{d}_{\text {up }}$ and $\mathrm{X}$-site orbital ) and spin polarized bandgap obtained from PBEsol $+\mathrm{U}$ are identical to $\mathrm{G}_{0} \mathrm{~W}_{0}$ calculation, thus the bandgap results of PBEsol+U are still acceptable. 
1. Kresse, G. \& Furthmuller, J. Efficient iterative schemes for ab initio total-energy calculations using a plane-wave basis set. Phys. Rev. B 54, 11169-11186, (1996)

2. Kresse, G. \& Furthmuller, J. Efficiency of ab-initio total energy calculations for metals and semiconductors using a plane-wave basis set. Computational Materials Science 6, 15-50, (1996).

3. Csonka, G.I., Perdew, J. P., Ruzsinszky, A., Philipsen, P. H. T., Lebegue, S., Paier. J., Vydrov, O. A., \& Ángyán, J. G. Generalized gradient approximation made simple. Phys. Rev. B. 79, 155107, (2009).

4. Blochl, P. E. Projector augmented-wave method. Phys. Rev. B 50, 17953-17979, (1994).

5. Grimme, S., Antony, J., Ehrlich, S. \& Krieg, H. A consistent and accurate ab initio parametrization of density functional dispersion correction (DFT-D) for the 94 elements H-Pu. J. Chem. Phys. 132, 154104, (2010).

6. Monkhorst, H. J. \& Pack, J. D. Special points for Brillouin-zone integrations. Phys. Rev. B 13, 5188-5192, (1976).

7. Liechtenstein, A. I., Anisimov, V. I. \& Zaanen, J. Density-functional theory and strong interactions: Orbital ordering in MottHubbard insulators. Phys. Rev. B 52, R5467 (1995)

8. Wegkamp, D., et al. Instantaneous bandgap collapse in photoexcited monoclinic $\mathrm{VO}_{2}$ due to photocarrier doping. Phys. Rev. Lett. 113 (21), 216401, (2014)

9. Aguado, F.; Rodríguez, F.; Valiente, R.; Itiè, J.-P.; Hanfland, M., Pressure effects on Jahn-Teller distortion in perovskites: The roles of local and bulk compressibilities. Phys. Rev. B 2012, 85 (10), 100101 